\title{
Time trends of clinical characteristics in hepatocellular carcinoma patients with chronic hepatitis $B$ virus infection: A field survey between 2000 and 2012
}

\author{
KEISUKE AMANO ${ }^{1,2}$, TAKUMI KAWAGUCHI ${ }^{1,3}$, RYOKO KUROMATSU ${ }^{1}$, ATSUSHI KAWAGUCHI ${ }^{4}$, \\ ICHIRO MIYAJIMA $^{1,2}$, TATSUYA IDE ${ }^{1,2}$, TATSUYUKI KAKUMA ${ }^{5}$ and MICHIO SATA ${ }^{1-3}$ \\ ${ }^{1}$ Division of Gastroenterology, Department of Medicine, Kurume University School of Medicine; \\ ${ }^{2}$ Consulting and Support Center for Liver Disease, Kurume University Hospital, ${ }^{3}$ Department of Digestive Disease \\ Information and Research, Kurume University School of Medicine, Kurume, Fukuoka 830-0011; \\ ${ }^{4}$ Department of Biomedical Statistics and Bioinformatics, Graduate School of Medicine, \\ Kyoto University, Kyoto, Kyoto 606-8501; ${ }^{5}$ Biostatistics Center, Kurume University, \\ Kurume, Fukuoka 830-0011, Japan
}

Received March 13, 2014; Accepted May 12, 2014

DOI: $10.3892 / \operatorname{mco} .2014 .398$

\begin{abstract}
The hepatitis $\mathrm{B}$ virus (HBV) carrier rate has decreased in Japan; however, the incidence of HBV infection among hepatocellular carcinoma (HCC) patients has not decreased accordingly. In this study, we aimed to assess the time trends of the clinical characteristics in $\mathrm{HCC}$ patients with chronic HBV infection. Between 2000 and 2012, we enrolled a total of $156 \mathrm{HCC}$ patients with chronic HBV infection in our field survey. The HCC risk was evaluated using the HCC prediction score, which is constructed from the characteristics of age, presence of liver cirrhosis and serum levels of albumin, bilirubin and HBV DNA. Lifestyle factors and the presence of diabetes mellitus were also evaluated. The time trends of patient characteristics were analyzed using the Jonckheere-Terpstra proportion trend test. Among HCC patients with chronic HBV infection, the proportion of patients at high risk according to the HCC prediction score significantly decreased during the study period $(\mathrm{P}=0.0005)$. Similarly, the proportion of patients with liver cirrhosis, $\leq 3.5 \mathrm{~g} / \mathrm{dl}$ serum albumin level, $>4 \log$ copies/ml serum HBV DNA level and $\geq 60 \mathrm{~g} /$ day alcohol intake
\end{abstract}

Correspondence to: Dr Takumi Kawaguchi, Division of Gastroenterology, Department of Medicine, Kurume University School of Medicine, 67 Asahi-machi, Kurume, Fukuoka 830-0011, Japan

E-mail: takumi@med.kurume-u.ac.jp

Abbreviations: $\mathrm{HBV}$, hepatitis $\mathrm{B}$ virus; $\mathrm{HCC}$, hepatocellular carcinoma; HB, hepatitis B; HBsAg, HB surface antigen; BMI, body mass index; WBC, white blood cell; AST, aspartate aminotransferase; ALT, alanine aminotransferase; AFP, $\alpha$-fetoprotein; DCP, des- $\gamma$-carboxy prothrombin; APRI, AST-to-platelet ratio index

Key words: chronic hepatitis B, liver cancer, time trend, aging, glucose metabolism disorder were also significantly decreased. The proportion of male and obese patients was not significantly altered, whereas the proportion of elderly ( $\geq 65$ years) and diabetic patients tended to increase during the study period $(\mathrm{P}=0.0654$ and $\mathrm{P}=0.0528$, respectively). In this study, we analyzed the time trends of the clinical characteristics in HCC patients with chronic HBV infection and demonstrated that aging and diabetes mellitus may be involved in the hepatocarcinogenesis in patients with chronic HBV infection.

\section{Introduction}

Hepatitis B virus (HBV) infection is a major cause of hepatocellular carcinoma (HCC) worldwide (1). In Japan, hepatitis B (HB) immunoglobulin and HB vaccination have been used since 1986 to prevent maternal-fetal transmission of HBV. As a result, the incidence of $\mathrm{HB}$ surface antigen (HBsAg) positivity among blood donors had decreased to $0.04 \%$ by 2006 , indicating the effectiveness of this program of vertical HBV transmission prevention (2). In addition, the first-generation nucleot(s)ide analogues were approved in 2000 and their long-term administration reduced the risk of HCC in patients with chronic HBV infection $(3,4)$. Thus, a reduction in the number of HCC patients with chronic HBV infection is to be expected. However, $16 \%$ of HCC patients are HBsAg-positive and the prevalence of HCC among patients with chronic HBV infection has not decreased in Japan since 1996 (5).

Several factors are associated with the development of HCC in patients with chronic HBV infection. Hepatic inflammation and fibrosis are well-documented risk factors (6). In addition, elevated serum HBV DNA level, HBV genotype $\mathrm{C}$ and a high serum HBsAg level are risk factors for $\operatorname{HCC}(7,8)$. Using a combination of these risk factors, several HCC prediction scores have been developed for HBV carriers $(9,10)$. The HCC prediction score reported by Wong et al (9) is a simple scoring system that uses parameters such as age, serum albumin level, serum bilirubin level, HBV DNA level and presence of liver 
cirrhosis, all of which are available in routine clinical practice and for field surveys.

Besides hepatic and viral factors, there are several other risk factors for the development of HCC. Lifestyle factors, such as alcohol intake and smoking, were shown to increase the risk for HCC (11). Obesity and type 2 diabetes mellitus with insulin resistance are also considered as risk factors $(12,13)$. The percentage of patients diagnosed with obesity- or type 2 diabetes mellitus-associated HCC has been increasing annually in Japan $(5,14)$. Moreover, elderly patients are at higher risk for developing HCC, even after being stratified by stage of hepatic fibrosis (15). Aging is a social issue in Japan and is becoming one of the major risk factors for HCC (15). Although all the abovementioned risk factors are considered to be independently associated with the onset of HCC, the time trends for these risk factors remain unclear in HCC patients with chronic HBV infection.

The aim of this study was to assess the time trends of clinical characteristics in HCC patients with chronic HBV infection.

\section{Subjects and methods}

Study design and subjects. We performed a field survey to assess the time trends of clinical characteristics in HCC patients with chronic HBV infection who visited Kurume University School of Medicine. The inclusion criteria included presence of HCC, HBsAg positivity and hepatitis C virus (HCV) antibody negativity. Patients with autoimmune hepatitis, primary biliary cirrhosis or hemochromatosis were excluded.

For evaluating the time trends for the annual number of HCC patients with HBV infection, we enrolled a total of 264 patients between 1991 and 2012. In Japan, nucleot(s)ide analogues were approved in 2000 and their use has markedly improved the natural course of patients with chronic hepatitis B. Therefore, the time trends of clinical characteristics were evaluated using $156 \mathrm{HCC}$ patients with chronic HBV infection between 2000 and 2012.

Clinical characteristics and lifestyle. Data on clinical characteristics and lifestyle factors, including age, gender, alcohol intake and cumulative cigarette consumption, were collected at the time of the first medical examination. Body mass index (BMI) was calculated as body weight in kilograms divided by the square of height in meters $\left(\mathrm{kg} / \mathrm{m}^{2}\right)$. The cumulative cigarette consumption was estimated using the Brinkman index (number of cigarettes smoked/day x years of smoking) (16).

Biochemical examinations. Venous blood samples were collected in the morning after a 12-h overnight fast. The following biochemical examinations were then performed, using standard clinical methods as previously described (17): blood platelet count, white blood cell count, prothrombin time, plasma glucose levels, hemoglobin A1c (HbA1c) levels and serum levels of aspartate aminotransferase (AST), alanine aminotransferase (ALT), albumin, total bilirubin, insulin, $\alpha$-fetoprotein (AFP) and des- $\gamma$-carboxy prothrombin (DCP).

Diagnosis of HCC. The diagnosis of HCC was based on the criteria of the clinical practice manual proposed by the Japan
Society of Hepatology (JSH) (18) using serum AFP and DCP levels and imaging techniques including ultrasonography, computed tomography, magnetic resonance imaging, hepatic angiography and/or tumor biopsy.

Staging and treatment of HCC. The Milan criteria (single nodule $\leq 5 \mathrm{~cm}$ or 3 nodules $<3 \mathrm{~cm}$ ) were used for HCC staging (14). The treatment of HCC was categorized as follows: i) local treatment such as hepatic resection, percutaneous ethanol injection therapy and radiofrequency ablation; ii) transcatheter arterial chemoembolization; iii) hepatic arterial infusion chemotherapy; iv) best supportive care; and v) liver transplantation.

HCC surveillance. The JSH proposed the Consensus-Based Clinical Practice Guidelines for the Management of HCC in Japan (18). These guidelines recommend assessing serum tumor marker levels and conducting imaging examinations, including ultrasonography, at intervals of 3-6 months, according to the HCC risk.

The patients were classified into the following $3 \mathrm{HCC}$ surveillance categories: i) patients with no HCC surveillance; ii) irregular surveillance (patients under HCC surveillance with either serum tumor marker levels or imaging examination, or at longer intervals than recommended); and iii) regular surveillance (patients under HCC surveillance according to the guidelines).

Measurement of HBsAg, anti-HCV antibodies and HBV DNA. Serum HBsAg and anti-HCV antibody levels were measured using standard clinical methods (Department of Clinical Laboratory Medicine, Kurume University Hospital, Kurume, Fukuoka, Japan) (19). The serum HBV DNA levels were measured using the COBAS TaqMan ${ }^{\circledR}$ HBV Auto v2.0 kit (Roche Diagnostics, Tokyo, Japan), which has a lower detection limit of $2.1 \log$ copies $/ \mathrm{ml}$.

Diagnosis of liver cirrhosis. Hepatic fibrosis was evaluated using the AST-to-platelet ratio index (APRI), as follows: serum AST level (U/l)/upper limit of normal AST level (33 U/1) x 100/platelet count (x10 /ml) (20). Patients with APRI values $>1.5$ were diagnosed with liver cirrhosis, as previously described (20).

Clinical scoring system for the prediction of $\mathrm{HCC}$ in chronic $H B V$ carriers. We employed the HCC prediction score reported by Wong et al (9). This score is constructed from the following 5 parameters: age ( $>50$ years, +3 points); serum albumin level ( $\leq 3.5 \mathrm{~g} / \mathrm{dl},+20$ points); serum bilirubin level (>1.05 mg/dl, +1.5 points); serum HBV DNA level (4-6 log copies/ml, +1 point, $>6 \log$ copies $/ \mathrm{ml},+4$ points); and liver cirrhosis (present, +15 points) (9). According to the total score, the patients were classified as having low $(<5)$, medium (5-19), or high risk (>19), as previously described (9).

Diagnosis of diabetes mellitus. Diabetes mellitus was diagnosed if the fasting blood glucose levels were $>126 \mathrm{mg} / \mathrm{dl}$ or the HbA1c levels were $>6.9 \%$, according to the Diagnostic Criteria for Diabetes Mellitus (21), or by the documented use of antidiabetic agents. 


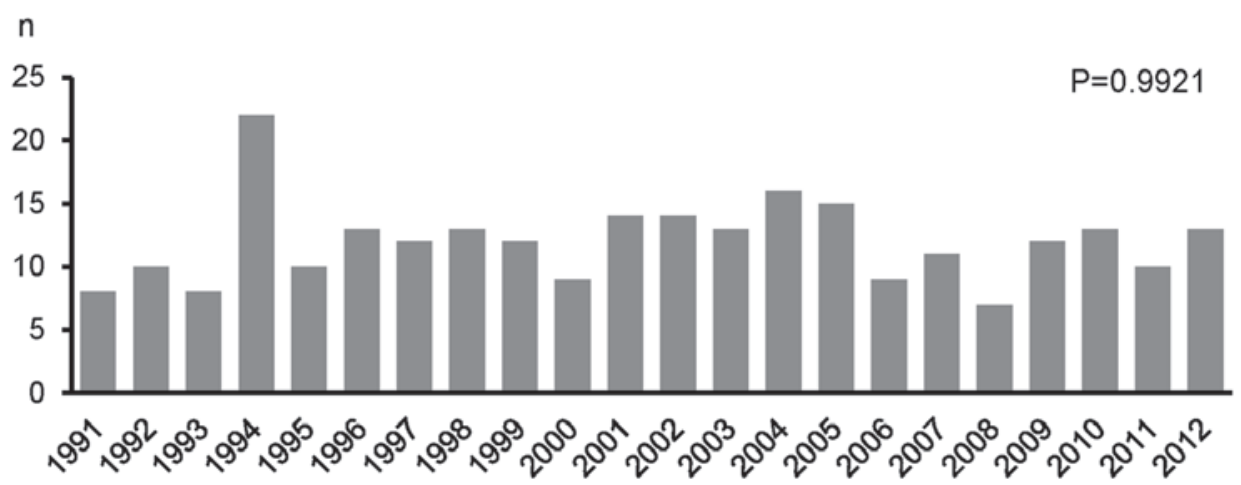

Figure 1. Time trends for the annual number of hepatocellular carcinoma patients with hepatitis B surface antigen positivity. The alterations in the number of patients were statistically evaluated by regression analysis. $\mathrm{P}<0.05$ was considered to indicate a statistically significant difference.

Statistical analysis. The alterations in the annual number of HCC patients with chronic HBV infection were statistically evaluated using regression analysis and the time trends of the patient characteristics were analyzed by the Jonckheere-Terpstra proportion trend test. The quoted $\mathrm{P}$-values are two-sided and $\mathrm{P}<0.05$ was considered to indicate a statistically significant difference. All the analyses were performed using SAS software, version 9.3 (SAS Institute Inc., Cary, NC, USA).

\section{Results}

Number of patients. The annual number of HCC patients with chronic HBV infection in the years between 1991 and 2012 is shown in Fig. 1. There was no significant decrease in the annual number of patients during the study period $(\mathrm{P}=0.9921$; $95 \%$ confidence interval, -0.23677 to 0.23451 ).

HCC prediction score. The proportion of patients with high and medium risk, according to the HCC prediction score, was significantly decreased, while the proportion of low-risk patients increased significantly during the study period $(\mathrm{P}=0.0005)$ (Fig. 2A).

The HCC prediction score is constructed using parameters such as age, serum albumin level, serum bilirubin level, serum HBV DNA level and presence of liver cirrhosis. Although the proportion of patients who were older than the threshold for increased risk ( $>50$ years) did not significantly change $(\mathrm{P}=0.1612)$ (Fig. $2 \mathrm{~B})$, the proportion of patients with serum albumin levels below the threshold for increased risk ( $\leq 3.5 \mathrm{~g} / \mathrm{dl}$ ) was significantly decreased $(\mathrm{P}=0.0142)$ (Fig. 2C). The proportions of patients with elevated serum bilirubin levels $(>1.05 \mathrm{mg} / \mathrm{dl})$ did not significantly change $(\mathrm{P}=0.2433)$ (Fig. 2D); however, the proportion of those with elevated HBV DNA levels ( $>4 \log$ copies/ml) and those with liver cirrhosis was significantly decreased during the study period ( $\mathrm{P}=0.0072)$ (Fig. 2E) and ( $\mathrm{P}=0.0001)$ (Fig. 2F).

Age and gender. The proportion of elderly patients ( $\geq 65$ years) exhibited a tendency to increase during the study period $(\mathrm{P}=0.0654)$ (Fig. 3A), whereas no significant change was observed in the proportion of male patients $(\mathrm{P}=0.3497)$ (Fig. 3B).
Alcohol intake and cumulative cigarette consumption. The proportion of patients with a habitual alcohol intake of $\geq 60 \mathrm{~g}$ /day significantly decreased during the study period $(\mathrm{P}=0.0408)$ (Fig. 4A). By contrast, no significant change was observed in the proportion of patients with a high Brinkmann index (>400; $\mathrm{P}=0.2095)$ (Fig. 4B).

Obesity and diabetes mellitus. No significant change was observed in the proportion of obese patients (BMI $\geq 25 \mathrm{~kg} / \mathrm{m}^{2}$ ) during the study period $(\mathrm{P}=0.5387)$ (Fig. 5A). However, the proportion of patients with diabetes mellitus exhibited a tendency to increase $(\mathrm{P}=0.0528)$ (Fig. 5B).

Stage, treatment and surveillance of HCC. A significant increase was observed in the proportion of HCC patients who met the Milan criteria during the study period $(\mathrm{P}=0.0212)$ (Fig. 6A). A significant increase was also observed in the proportion of patients receiving local treatment during the study period $(\mathrm{P}=0.0488)$ (Fig. 6B). The proportion of patients who were followed up with regular surveillance also increased significantly during the study period $(\mathrm{P}=0.0283)$ (Fig. 6C).

\section{Discussion}

In this study, we demonstrated that the annual number of HCC patients with chronic HBV infection did not significantly decrease, although the patient characteristics were significantly altered between 2000 and 2012 in Japan. The proportion of HCC patients in the high-risk group of the HCC prediction score was significantly decreased, whereas the proportion of elderly patients and patients with diabetes mellitus exhibited a tendency to increase. We also demonstrated that the proportion of HCC patients who met the Milan criteria and those who received local treatment increased significantly during the study period.

We observed that the annual number of HCC patients with chronic HBV infection did not decrease between 1991 and 2012. We had previously reported that the incidence of $\mathrm{HBsAg}$ positivity among $\mathrm{HCC}$ patients did not change in a Japanese cohort study conducted between 1996 and 2008 (5). The HCC prediction score classifies the risk of HCC in HBV carriers as low, medium and high risk (9). We demonstrated that the 

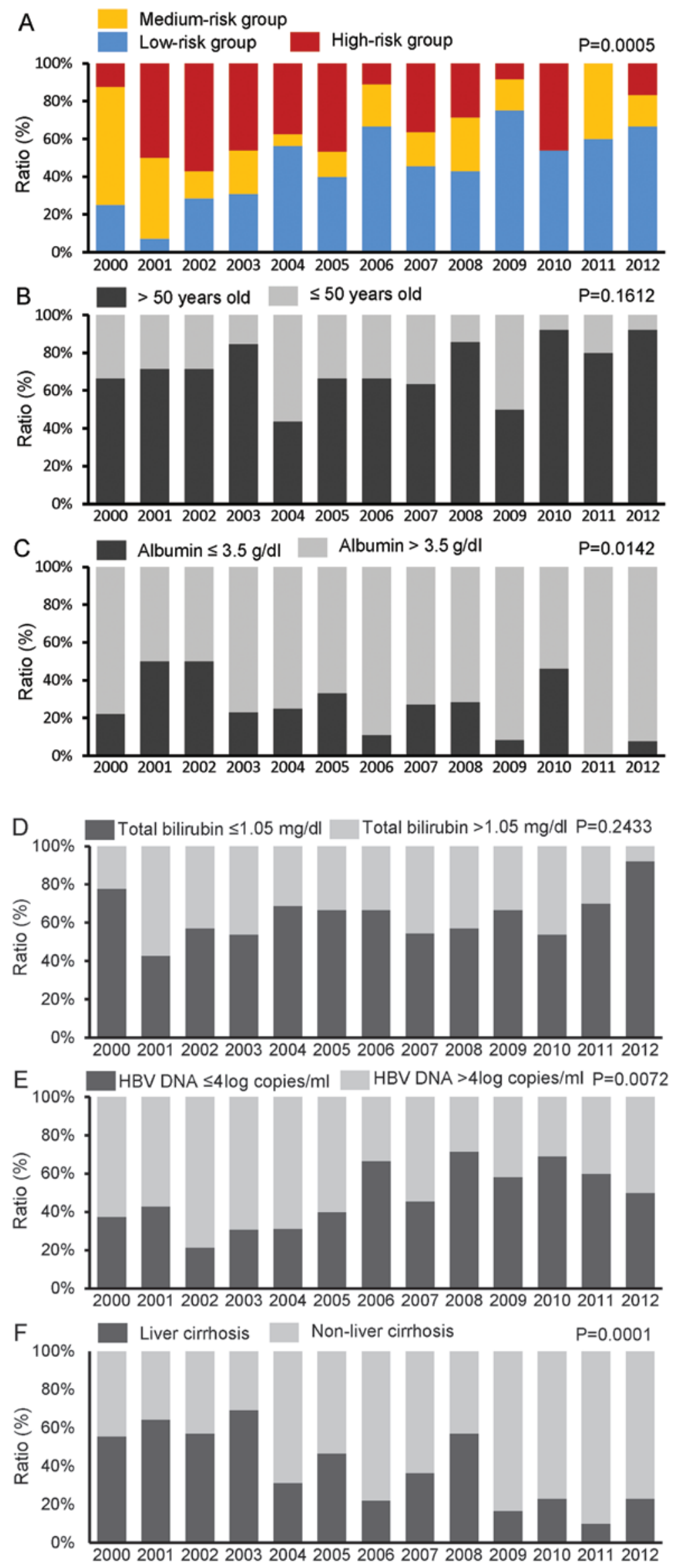

Figure 2. Time trends for the proportion of (A) patients at high risk according to the hepatocellular carcinoma prediction score, (B) patients aged $>50$ years, (C) patients with serum albumin levels $\leq 3.5 \mathrm{~g} / \mathrm{dl}$, (D) patients with serum bilirubin levels $>1.05 \mathrm{mg} / \mathrm{dl}$, (E) patients with serum hepatitis B virus (HBV) DNA level $>4 \log$ copies $/ \mathrm{ml}$ and $(\mathrm{F})$ patients with liver cirrhosis. The time trends of patient characteristics were analyzed by the Jonckheere-Terpstra proportion trend test. $\mathrm{P}<0.05$ was considered to indicate a statistically significant difference. 

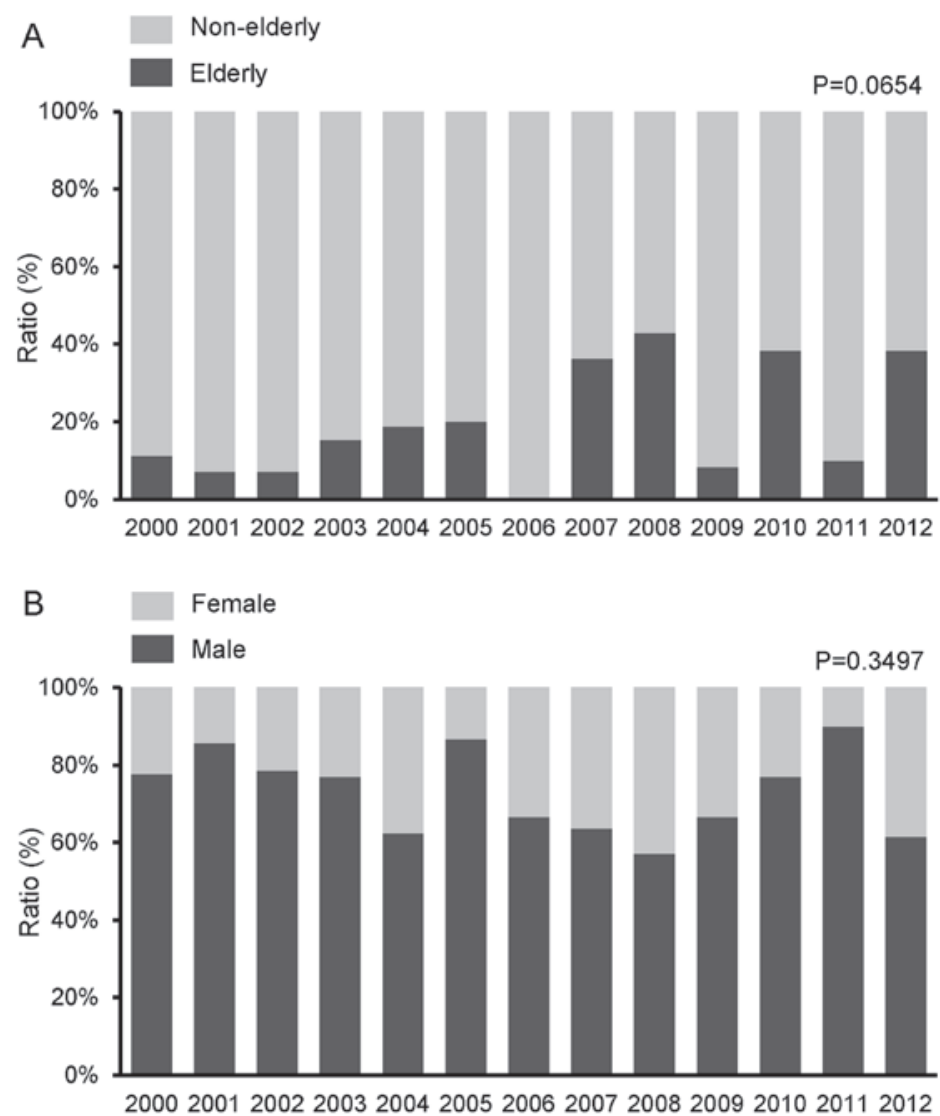

Figure 3. Time trends for the proportion of (A) elderly and (B) male patients. The time trends of patient characteristics were analyzed by the Jonckheere-Terpstra proportion trend test. $\mathrm{P}<0.05$ was considered to indicate a statistically significant difference.
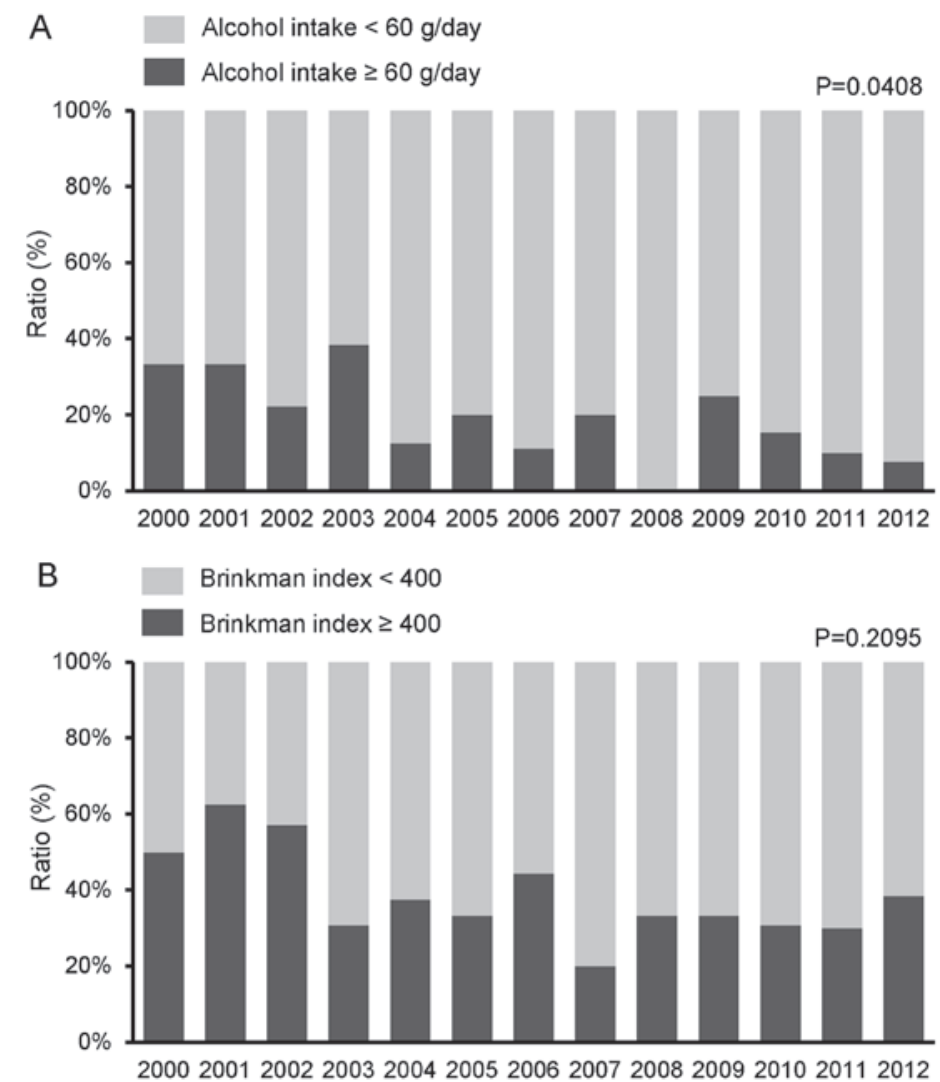

Figure 4. Time trends for the proportion of (A) patients with an alcohol intake $\geq 60 \mathrm{~g} /$ day and (B) patients with a Brinkman index $\geq 400$. The time trends of patient characteristics were analyzed by the Jonckheere-Terpstra proportion trend test. $\mathrm{P}<0.05$ was considered to indicate a statistically significant difference. 

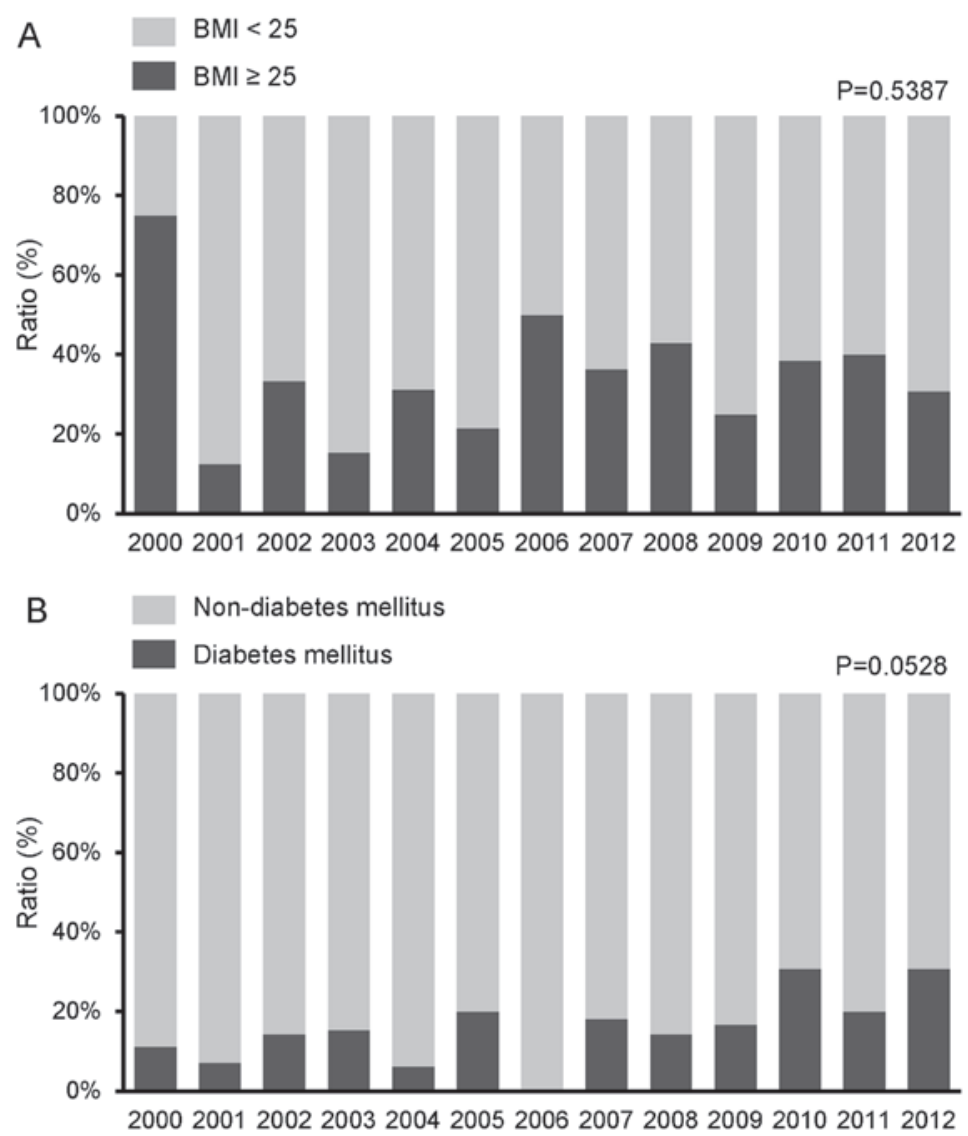

Figure 5. Time trends for the proportion of (A) patients with a body mass index (BMI) $\geq 25 \mathrm{~kg} / \mathrm{m}^{2}$ and (B) patients with diabetes mellitus. The time trends of patient characteristics were analyzed by the Jonckheere-Terpstra proportion trend test. $\mathrm{P}<0.05$ was considered to indicate a statistically significant difference.

proportion of patients at high risk for HCC was significantly decreased during the study period. The estimation of the HCC prediction score is based on age, serum albumin level, serum bilirubin level, HBV DNA level and the presence of liver cirrhosis. We observed significant decreases for each factor, except for age and serum bilirubin level, in the proportion of patients who met the criteria for increased HCC risk. Thus, liver function is currently better preserved and HBV DNA levels are more efficiently controlled in HCC patients with chronic HBV infection. Taken together, these data suggest that, besides HBV-related viral and hepatic factors, other risk factors may play significant roles in HCC patients with chronic HBV infection.

Aging, gender and lifestyle are involved in the development of HCC. In this study, the proportion of male patients and of patients with a high Brinkman index was unchanged during the study period and the proportion of habitual drinkers was decreased. By contrast, the proportion of elderly patients exhibited a tendency to increase during the study period. It was recently established that elderly patients with $\mathrm{HCV}$ are at higher risk for $\mathrm{HCC}$ and that eradication of $\mathrm{HCV}$ exerts a less significant preventive effect on hepatocarcinogenesis in elderly patients (15). Moreover, hepatocyte aging, as assessed by relative nuclear size, was found to be associated with hepatocarcinogenesis in patients with non-HBV non-HCV non-alcoholic chronic liver injury (22). Although the mechanisms underlying age-related hepatocarcinogenesis have not been fully elucidated, aging is associated with telomere shortening, which in turn results in chromosomal instability and the inactivation of cell cycle checkpoints $(23,24)$. In addition, aging is known to enhance DNA hypermethylation of tumor suppressor genes, which is associated with the onset of HCC (25). Thus, our findings, along with those of previous clinical and basic research, suggest that aging may be an significant factor in HCC patients with chronic HBV infection.

The proportion of patients with diabetes mellitus exhibited a tendency to increase during the study period. However, Chen et al (26) reported that diabetes mellitus was not found to be a risk factor for HCC in an HBV- and HCV-endemic area of Southern Taiwan. The reasons for the discrepancy between our findings and theirs remain unclear; however, it should be noted that diabetes mellitus was defined only by the presence of a fasting blood glucose level $>126 \mathrm{mg} / \mathrm{dl}$ in the study by Chen et al (26). Since fasting blood glucose levels are lower in patients with chronic liver disease, owing to the depletion of hepatic glycogen stores, it is possible that the number of patients with diabetes mellitus was underestimated in the study of Chen et al (26). In HBV carriers, diabetes mellitus was reported to be an independent risk factor for HCC by large-scale prospective studies $(27,28)$. Moreover, HCC was shown to be a major cause of death among patients with diabetes mellitus in Japan (29). Furthermore, a characteristic of diabetic patients with chronic liver disease is hyperinsulinemia $(30,31)$. Insulin is known to bind both insulin and insulin-like growth factor-1 (IGF-1) receptors and to activate cell mitogenic and growth pathways $(13,32)$. Insulin also binds to IGF-binding protein, 


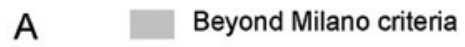

Within Milano criteria

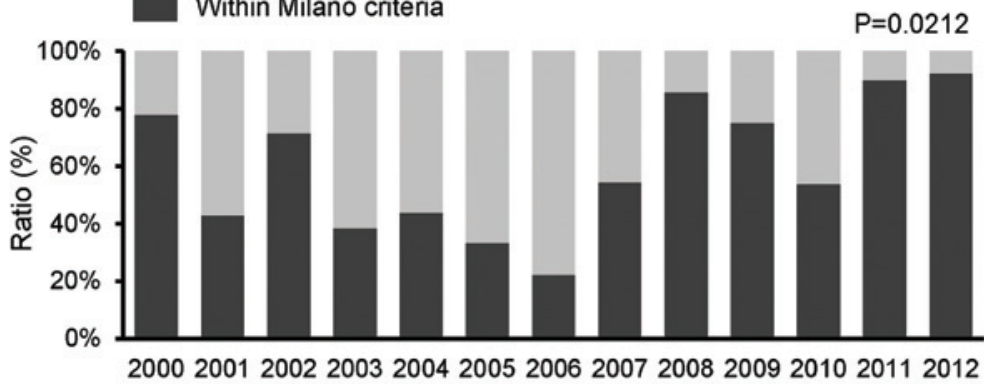
B Local treatment
TACE

Hepatic arterial infusion chemotherapy
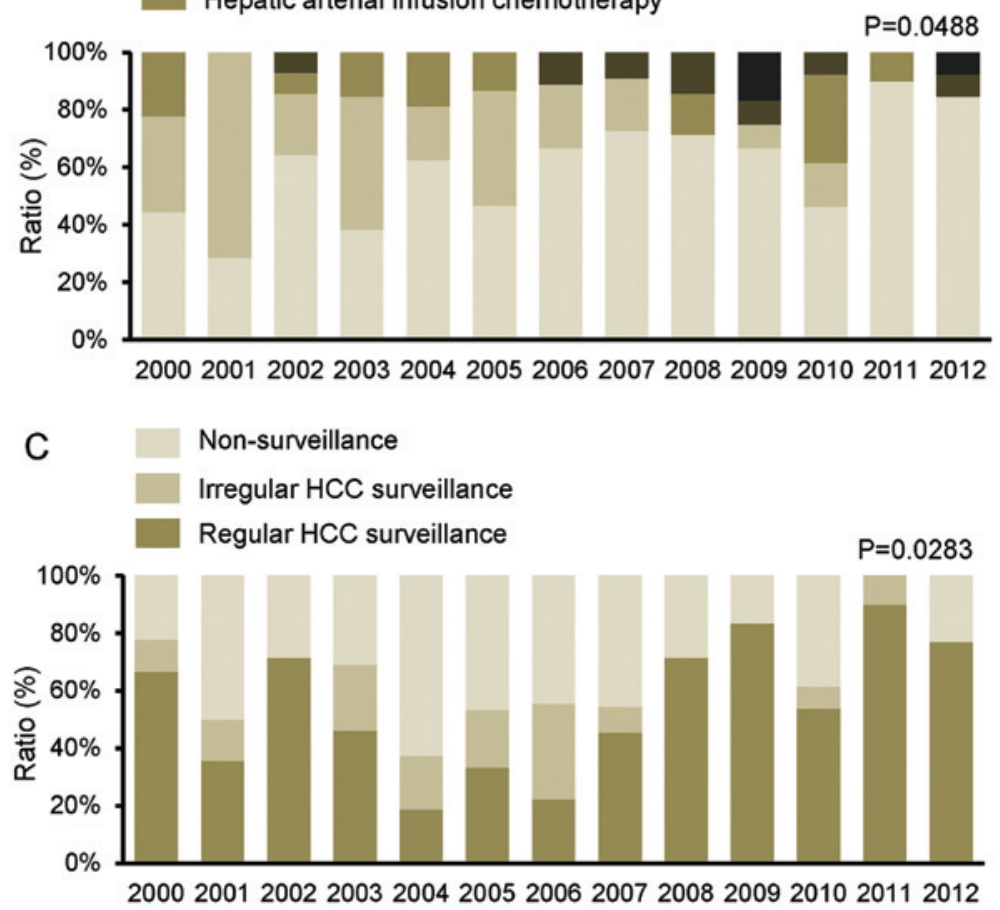

Figure 6. Time trends for the proportion of (A) patients who met the Milan criteria, (B) patients treated with local therapy and (C) patients who received regular follow-up surveillance. The time trends of patient characteristics were analyzed by the Jonckheere-Terpstra proportion trend test. $\mathrm{P}<0.05$ was considered to indicate a statistically significant difference. TACE, transcatheter arterial chemoembolization; HCC, hepatocellular carcinoma.

leading to an increase in the serum levels of IGF-1 $(13,32)$. Thus, diabetes mellitus with hyperinsulinemia is considered to accelerate hepatocarcinogenesis.

We also demonstrated that the proportion of HCC patients who met the Milan criteria and of those who received local treatment increased significantly during the study period. Although this study was not intended to determine the cause for the increase in early detection rates of HCC and treatment with local therapy, the sensitivity and accuracy of DCP and lens culinaris agglutinin-reactive fraction of AFP diagnostic systems have been improved (33). The diagnostic ability of magnetic resonance imaging for HCC has significantly improved through the use of gadolinium ethoxybenzyl diethylenetriamine pentaacetic acid (34). Hepatic fibrosis and liver function have also improved after the introduction of nucleot(s)ide analogues $(35,36)$. Radiofrequency ablation therapy has supplanted ethanol injection therapy and has allowed for the treatment of HCCs of greater dimensions (37). In addition, the proportion of patients who were followed up with regular surveillance increased significantly during the study period. Surveillance is an important factor for the early detection of HCC $(38,39)$. Taken together, these facts suggest that the increase in the rate of early detection and the treatment with local therapy is likely due to the recent advances in HCC diagnostic and treatment methodologies, as well as the improvements in the management of $\mathrm{HBV}$-infected patients.

In conclusion, we demonstrated that there were significant alterations in the clinical characteristics of $\mathrm{HCC}$ patients with chronic HBV infection between 2000 and 2012. The proportion of HCC patients with conventional risk factors, such as liver cirrhosis and high serum HBV DNA levels, decreased significantly. By contrast, the proportion of elderly patients and patients with diabetes mellitus exhibited a tendency to increase. Moreover, we demonstrated that the proportion of HCC patients who met the Milan criteria and of those who underwent local treatment increased significantly during the study period. This field survey provides important information 
that may be used when developing strategies for improving the outcome of HCC patients with chronic HBV infection in the era of nucleot(s)ide analogue therapy.

\section{Acknowledgements}

This study was supported, in part, by a Grant-in-Aid for Scientific Research (C) (no. 21590865 to M.S.) from the Ministry of Education, Culture, Sports, Science and Technology of Japan and by Health and Labour Sciences Research Grants for Research on Hepatitis from the Ministry of Health, Labour and Welfare of Japan (H24-Hepatitis-General-007).

\section{References}

1. El-Serag HB: Epidemiology of viral hepatitis and hepatocellular carcinoma. Gastroenterology 142: 1264-1273 e1261, 2012.

2. Yoshikawa A, Suzuki K, Abe A, et al: Effect of selective vaccination on a decrease in the rate of hepatitis B virus-positive Japanese first-time blood donors. Transfus Med 19: 172-179, 2009.

3. Hosaka T, Suzuki F, Kobayashi M, et al: Long-term entecavir treatment reduces hepatocellular carcinoma incidence in patients with hepatitis B virus infection. Hepatology 58: 98-107, 2013.

4. Matsumoto A, Tanaka E, Rokuhara A, et al; Inuyama Hepatitis Study Group: Efficacy of lamivudine for preventing hepatocellular carcinoma in chronic hepatitis B: A multicenter retrospective study of 2795 patients. Hepatol Res 32: 173-184, 2005.

5. Taura N, Fukushima N, Yastuhashi H, et al: The incidence of hepatocellular carcinoma associated with hepatitis $\mathrm{C}$ infection decreased in Kyushu area. Med Sci Monit 17: PH7-PH11, 2011.

6. McMahon BJ: The natural history of chronic hepatitis B virus infection. Hepatology 49 (Suppl 5): S45-S55, 2009.

7. Chen CJ, Yang HI, Su J, et al; REVEAL-HBV Study Group: Risk of hepatocellular carcinoma across a biological gradient of serum hepatitis B virus DNA level. JAMA 295: 65-73, 2006.

8. Kao JH, Chen PJ, Lai MY and Chen DS: Hepatitis B genotypes correlate with clinical outcomes in patients with chronic hepatitis B. Gastroenterology 118: 554-559, 2000.

9. Wong VW, Chan SL, Mo F, et al: Clinical scoring system to predict hepatocellular carcinoma in chronic hepatitis B carriers. J Clin Oncol 28: 1660-1665, 2010.

10. Wong GL and Wong VW: Risk prediction of hepatitis B virus-related hepatocellular carcinoma in the era of antiviral therapy. World J Gastroenterol 19: 6515-6522, 2013.

11. Purohit V, Rapaka R, Kwon OS and Song BJ: Roles of alcohol and tobacco exposure in the development of hepatocellular carcinoma. Life Sci 92: 3-9, 2013.

12. Farrell G: Insulin resistance, obesity and liver cancer. Clin Gastroenterol Hepatol 12: 117-119, 2014.

13. Kawaguchi T, Izumi N, Charlton MR and Sata M: Branched-chain amino acids as pharmacological nutrients in chronic liver disease. Hepatology 54: 1063-1070, 2011.

14. Kawaguchi T, Kakuma T, Yatsuhashi H, et al: Data mining reveals complex interactions of risk factors and clinical feature profiling associated with the staging of non-hepatitis B virus/non-hepatitis $C$ virus-related hepatocellular carcinoma. Hepatol Res 41: 564-571, 2011.

15. Asahina $\mathrm{Y}$, Tsuchiya $\mathrm{K}$, Tamaki N, et al: Effect of aging on risk for hepatocellular carcinoma in chronic hepatitis $\mathrm{C}$ virus infection. Hepatology 52: 518-527, 2010.

16. Yamada S, Kawaguchi A, Kawaguchi T, et al: Serum albumin level is a notable profiling factor for non-B, non- $\mathrm{C}$ hepatitis virus-related hepatocellular carcinoma: A data-mining analysis. Hepatol Res: Jul 2, 2013 (Epub ahead of print). doi: 10.1111/hepr.12192.

17. Kawaguchi T, Shiba N, Maeda T, et al: Hybrid training of voluntary and electrical muscle contractions reduces steatosis, insulin resistance, and IL-6 levels in patients with NAFLD: a pilot study. J Gastroenterol 46: 746-757, 2011.

18. Kudo M, Izumi N, Kokudo N, et al; HCC Expert Panel of Japan Society of Hepatology: Management of hepatocellular carcinoma in Japan: Consensus-Based Clinical Practice Guidelines proposed by the Japan Society of Hepatology (JSH) 2010 updated version. Dig Dis 29: 339-364, 2011.
19. Kawaguchi T, Ide T, Taniguchi E, et al: Clearance of HCV improves insulin resistance, beta-cell function, and hepatic expression of insulin receptor substrate 1 and 2. Am J Gastroenterol 102: 570-576, 2007.

20. Wai CT, Greenson JK, Fontana RJ, et al: A simple noninvasive index can predict both significant fibrosis and cirrhosis in patients with chronic hepatitis C. Hepatology 38: 518-526, 2003.

21. Seino Y, Nanjo K, Tajima N, et al: Report of the Committee on the classification and diagnostic criteria of diabetes mellitus. Diabetol Int 1: 2-20, 2010.

22. Nakajima T, Nakashima T, Yamaoka J, et al: Greater age and hepatocellular aging are independent risk factors for hepatocellular carcinoma arising from non-B non-C non-alcoholic chronic liver disease. Pathol Int 61: 572-576, 2011.

23. Plentz RR, Schlegelberger B, Flemming P, et al: Telomere shortening correlates with increasing aneuploidy of chromosome 8 in human hepatocellular carcinoma. Hepatology 42: 522-526, 2005.

24. Plentz RR, Park YN, Lechel A, et al: Telomere shortening and inactivation of cell cycle checkpoints characterize human hepatocarcinogenesis. Hepatology 45: 968-976, 2007.

25. Wang Y, Cheng J, Xu C, et al: Quantitative methylation analysis reveals gender and age differences in p16INK4a hypermethylation in hepatitis B virus-related hepatocellular carcinoma. Liver Int 32: 420-428, 2012.

26. Chen CT, Chen JY, Wang JH, et al: Diabetes mellitus, metabolic syndrome and obesity are not significant risk factors for hepatocellular carcinoma in an HBV- and HCV-endemic area of Southern Taiwan. Kaohsiung J Med Sci 29: 451-459, 2013.

27. Gao C, Zhao HC, Li JT and Yao SK: Diabetes mellitus and hepatocellular carcinoma: comparison of Chinese patients with and without HBV-related cirrhosis. World J Gastroenterol 16: 4467-4475, 2010.

28. Chen CL, Yang HI, Yang WS, et al: Metabolic factors and risk of hepatocellular carcinoma by chronic hepatitis B/C infection: a follow-up study in Taiwan. Gastroenterology 135: 111-121, 2008.

29. Hotta N, Nakamura J, Iwamoto Y, Ohno Y, Kasuga M, Kikkawa R and Toyota T: Causes of death in Japanese diabetics: A questionnaire survey of 18,385 diabetics over a 10 -year period. J Diabetes Investig 1: 66-76, 2010.

30. Eslam M, Aparcero R, Kawaguchi T, Del Campo JA, Sata M, Khattab MA and Romero-Gomez M: Meta-analysis: insulin resistance and sustained virological response in hepatitis $\mathrm{C}$. Aliment Pharmacol Ther 34: 297-305, 2011.

31. Kawaguchi T, Yoshida T, Harada M, et al: Hepatitis C virus down-regulates insulin receptor substrates 1 and 2 through up-regulation of suppressor of cytokine signaling 3. Am J Pathol 165: 1499-1508, 2004.

32. Calle EE and Kaaks R: Overweight, obesity and cancer: epidemiological evidence and proposed mechanisms. Nat Rev Cancer 4: 579-591, 2004.

33. Kobayashi M,Hosaka T,Ikeda K, et al: Highly sensitive AFP-L3\% assay is useful for predicting recurrence of hepatocellular carcinoma after curative treatment pre- and postoperatively. Hepatol Res 41: 1036-1045, 2011.

34. Inoue $\mathrm{T}$, Kudo $\mathrm{M}$, Komuta $\mathrm{M}$, et al: Assessment of Gd-EOB-DTPA-enhanced MRI for HCC and dysplastic nodules and comparison of detection sensitivity versus MDCT. J Gastroenterol 47: 1036-1047, 2012.

35. Koga H, Ide T, Oho K, et al: Lamivudine treatment-related morphological changes of esophageal varices in patients with liver cirrhosis. Hepatol Res 37: 503-509, 2007.

36. Chang TT, Liaw YF, Wu SS, et al: Long-term entecavir therapy results in the reversal of fibrosis/cirrhosis and continued histological improvement in patients with chronic hepatitis B. Hepatology 52: 886-893, 2010.

37. Tateishi R, Shiina S, Teratani T, et al: Percutaneous radiofrequency ablation for hepatocellular carcinoma. An analysis of 1000 cases. Cancer 103: 1201-1209, 2005.

38. Ando E, Kuromatsu R, Tanaka M, et al: Surveillance program for early detection of hepatocellular carcinoma in Japan: results of specialized department of liver disease. J Clin Gastroenterol 40: 942-948, 2006.

39. Nakano M, Ando E, Kuromatsu R, et al: Recent progress in the management of hepatocellular carcinoma detected during a surveillance program in Japan. Hepatol Res 40: 989-996, 2010. 